\title{
Las sociedades aprenden y aún así el mundo es difícil de cambiar
}

\author{
Klaus Eder ${ }^{1}$
}

\section{Resumen}

Evolución y aprendizaje son dos conceptos analíticamente distintos. Las personas aprenden y aún así la evolución ("el cambio") no ocurre necesariamente. Para clarificar este problema el concepto de aprendizaje es explicado. Un primer problema tratado es la pregunta acerca de quién está aprendiendo. Aquí se propone un desplazamiento desde la perspectiva del actor individual a una perspectiva de la interacción (utilizando los argumentos teóricos de Habermas y Luhmann para tal desplazamiento). Sin embargo, ambos idealizan las precondiciones que los interactuantes comparten mientras aprenden colectivamente. En contra de supuestos racionalistas, se argumenta que las personas para poder aprender necesitan compartir un mundo narrativo. ¿Qué aprenden? Adquieren conocimiento y aprenden a aprender. Esto aún no soluciona el problema de por qué aprenden.Yendo más allá de la idea de procesos de aprendizaje impulsados por el propio individuo, se identifica a las situaciones de incertidumbre como el mecanismo de aprendizaje. Las situaciones de interrupción de órdenes narrativos representarían la forma de incertidumbre más profunda para fomentar el aprendizaje. La conclusión es que el aprendizaje no garantiza la evolución. Sin embargo, provee las mutaciones para que los procesos evolutivos ocurran.

\section{Societies learn and yet the world is hard to change Abstract}

Evolution and learning are two analytically distinct concepts. People learn yet evolution ("change") does not necessarily take place. To clarify this problem the concept of learning is explicated. A first problem addressed is the question of who is learning. Here a shift from the single actor perspective to an interaction perspective is proposed (using Habermas and Luhmann as theoretical arguments for such a shift). Both however idealize he preconditions that interactants share while learning collectively. Against rationalist assumptions it is argued that in order to learn people need a narratively based shared world. What do they learn? They acquire knowledge and they learn how to learn. This still does not solve the problem why they learn. Going beyond the idea of self-propelling learning processes situations of uncertainty are identified as the mechanism of learning, naming situations of breakdown of narrative orders the deepest uncertainty to foster learning. Learning, this is the conclusion, does not guarantee evolution. It however provides the mutations for evolutionary processes to take place.

${ }^{1}$ Profesor de teoría sociológica de la Humboldt-Universität zu Berlin (keder@rz.hu-berlin.de). El siguiente artículo fue originalmente publicado en European Journal for Social Theory 2 (1999) y la presente traducción fue realizada por Mónica Gerber, Socióloga de la Universidad de Chile. 


\section{El problema teórico}

Las sociedades ciertamente evolucionan. Esta evolución se basa - a diferencia de la evolución natural que se basa en la evolución genética - en procesos "epigenéticos", los cuales llamamos evolución cultural. Epigénesis es un concepto que se refiere al aprendizaje como un mecanismo secundario de la evolución natural ${ }^{2}$. Con esto no se pretende decir que los animales no aprenden. Sí aprenden. Pero su aprendizaje no es constitutivo de la evolución de su especie.Vincular los mecanismos y lógicas de la evolución social a la evolución cultural significa que el aprendizaje de las sociedades es constitutivo de la evolución social. La evolución cultural es una teoría de la manera en que el aprendizaje procede ${ }^{3}$. Debido a esto, acentúo desde un principio que las sociedades sí aprenden y que el aprendizaje es incluso constitutivo de la evolución social.

El hecho de que las sociedades aprenden no fue tematizado hasta el comienzo de la sociedad moderna.Ya sea que lo asumamos o no como una ideología occidental, la evolución cultural se ha convertido en un tema de reflexión como tal, llegando a ser una idea específica acerca del aprendizaje que ha ocurrido a lo largo de la historia de la humanidad. Contrastar esto con la manera en que las sociedades tradicionales se han hecho cargo de este problema, podría darnos una primera idea acerca de la naturaleza de esta noción de aprendizaje, específicamente moderna.

Las sociedades tradicionales también tenían teorías acerca del rumbo de la historia, las cuales luego se convirtieron en efectivas en moldear el rumbo de la historia. Su idea era proponer un futuro estado hacia el cual se mueve la existencia humana en sociedad. En la religión occidental la idea es clara: se trata de un proceso que lleva a un mundo mejor bajo la guía de Dios. De esta manera, tenemos una explícita "teoría del aprendizaje", construida en un modo de mirar el mundo social: Dios como el educador del mundo social, el cual trata de aprender de la mejor manera que puede. Las religiones orientales, que se preocupan de la idea de perfección (como en el caso de las religiones indias) o de ideas acerca de convertirse en última instancia en idénticos con el cosmos, implican ideas de estados de perfección, lo cual es otro concepto de los procesos de aprendizaje. Especialistas, "virtuosi" religiosos (desde monjes budistas hasta curas védicos) ayudaban a lo largo de este proceso para llegar a estas etapas. Esta idea de proceso de aprendizaje no se refería simplemente a lo indi-

\footnotetext{
${ }^{2}$ Para este tipo de argumentos, ver especialmente Boyd/Richerson (1985). Los autores analizan las diferencias específicas entre la transmisión cultural y la transmisión genética y las funciones que los errores, la variación no azarosa y los efectos azarosos del medio ambiente tienen sobre los mecanismos de transmisión cultural. Para una versión anterior de tal argumento, ver Pringle (1951).

${ }^{3}$ Si el uso del término "evolución" en el concepto de evolución cultural es una buena elección puede ser cuestionado, dado que la evolución social y cultural son procesos totalmente diferentes. La evolución natural se basa en mecanismos genéticos (y a veces hablamos de manera laxa sobre evolución genética). De la misma manera laxa, hablamos de evolución social basada en mecanismos de aprendizaje (y luego, frecuentemente hablamos de evolución cultural como procesos de aprendizaje). Pero estos son problemas terminológicos. Para este problema ver a Boyd/Richerson (1985).
} 
vidual. Dado que estas ideas en las religiones de tipo oriental previeron que ningún individuo podía alcanzar la etapa de perfección en su vida, la idea de una serie de vidas fue desarrollada, la cual garantizaba que el aprendizaje continuaría aun mas allá de la mera vida de un individuo. Así, la unidad de aprendizaje ha sido el colectivo, para el cual la vida individual era el medio hacia la perfección de los seres humanos como tal ${ }^{4}$.

Este breve excurso sobre la historia cultural y religiosa (y habría mucho que agregar respecto a otras tradiciones religiosas) fue pensado como un contraste de lo que pasó en las sociedades modernas. Dicho de forma breve: la sociedad moderna remplazó a Dios como el educador del hombre. Remplazó a Dios con intelectuales, es decir, con la sociedad. De esta manera, la base del aprendizaje no se situaba más fuera de la sociedad, sino dentro de la sociedad.

Tenemos dos ejemplos principales de este nuevo tipo de auto-consciencia de la capacidad de aprendizaje de la sociedad. El primero es Marx y su idea del desarrollo de las fuerzas de producción, la cual es una teoría acerca de un proceso de aprendizaje técnico o cognitivo, permitiendo a los humanos controlar su medio ambiente y usarlo para sus propios fines. Esta idea no era particular de Marx. Él simplemente dio expresión a un sentimiento ampliamente extendido, de una sociedad moderna aprendiendo a una velocidad increíble - en ciencia, en tecnología, en la cantidad de conocimiento práctico necesario para transformar la naturaleza en bienes económicos. El segundo ejemplo es la idea de Herder de una "Erziehung des Menschengeschlechts", de una educación de la raza humana hacia la perfección moral, siendo los educadores los moralistas ilustrados de su tiempo y teniendo su apoteosis en la filosofia de la historia hegeliana. Ambas ideas terminan en la noción de que el aprendizaje es un proceso auto-regulado que ocurre dentro de la sociedad y que la mueve a través de etapas progresivas hacia el universalismo moral (esta ha sido la tradición ética) o hacia el dominio tecnológico de la naturaleza (esta ha sido la tradición cognitiva/científica).

La sociología ha terminado con la manera ingenua de mirar la sociedad. La sociología del conocimiento ha mostrado que esta idea de sociedad como una entidad que aprende es en sí misma una construcción social, constituyente de la cultura de la sociedad moderna. La sociedad aprendió que ella aprende.Y esto aumentó tremendamente la velocidad de la evolución cultural, o la velocidad del aprendizaje social ${ }^{5}$. Las sociedades modernas crearon así un nuevo tipo de conocimiento reflexivo acerca de la manera en que se observa a sí misma. Esta es la reflexividad de las sociedades modernas. La sociedad moderna es una sociedad que aprende. Todo esto se ha convertido en conocimiento común. Y sin embargo, aún no tenemos una explicación ${ }^{4} \mathrm{El}$ modo de la "revelación" es un buen indicador de las diferencias entre estas religiones.

${ }^{5}$ Esto es lo que los contemporáneos de la época de la Ilustración notaron cuando observaron sus propias sociedades: estaban irritados con todas las cosas nuevas por venir, teniendo la sensación de que mucho desorden es creado a través de mucho aprendizaje.Ver Eder (1985). 
de por qué toda esta reflexividad no ha ayudado a crear la sociedad que queremos. De esta manera, la idea de una teoría del aprendizaje social genera una paradoja: ¿por qué, a pesar de todos los tipos de aprendizaje, es tan dificil cambiar el mundo? Esta paradoja será tratada más adelante y eventualmente será resuelta a través de una teoría de aprendizaje social.

Para hacer esto, debo entender y clarificar cómo las sociedades aprenden, qué aprenden y por qué aprenden. Esta última pregunta es importante para explicar por qué las sociedades modernas a veces no usan lo que han aprendido y para explicar por qué en ciertos momentos bloquean los procesos de aprendizaje. La noción teórica de aprendizaje con la que contamos parece ser insuficiente para responder a tales preguntas. Por lo tanto, debo reconstruir primero lo que ha sido dicho acerca de los procesos de aprendizaje, ver donde se ha tomado la dirección equivocada y buscar vías alternativas para construir modelos teóricos de aprendizaje social, antes de empezar la discusión de por qué el aprendizaje a veces ocurre y a veces no.

\section{2. ¿Cómo aprenden las sociedades?}

\subsection{Actores que aprenden}

La primera opción que ha dirigido la modelación y teorización de los procesos de aprendizaje social tiene que ver con el supuesto de que para entender cómo aprende la sociedad, debemos entender como aprenden los individuos ${ }^{7}$. Este es el famoso problema de los micro-fundamentos de los macro-desarrollos, el problema de que la historia es el resultado de una multitud de acciones individuales ${ }^{8}$. Me gustaría argumentar que esta manera de enmarcar el problema implica un supuesto razonado inadecuadamente, el cual puede ser identificado al mirar modos individualistas de pensar un orden social.

La discusión teórica en sociología nos ofrece un modelo sofisticado para entender la base a nivel micro del macro-aprendizaje. Este es el modelo de socialización, la idea de que una sociedad socializa a sus miembros inculcando normas y valores. Este modelo de socialización ha sido prominente por largo tiempo, digamos desde Parsons hasta Habermas. El carácter específico de estas teorías es que relacionan una perspectiva sistémica con una perspectiva individualista del sujeto. Parsons ve la acción

\footnotetext{
${ }^{6}$ Esta pregunta forma parte del título de un interesante artículo de Peters (1994).

${ }^{7}$ Este es un tema clásico en la teoría de la evolución. Ver Fishbein (1976) o Plotkin (1982). En teoría social, psicólogos sociales han apoyado esta perspectiva. Un clásico al respecto es la obra de Bandura (1977) o más recientemente la de Holland et al. (1986).

${ }^{8}$ Esta discusión se ha desarrollado de manera muy diferenciada. Para una buena visión general ver a Alexander et al. (1987).

${ }^{9}$ Esta discusión se encuentra resumida en sí misma en Habermas (1987).
} 
como gobernada por cuatro "pattern-variables", las cuales son parte de un sistema de acción. Para nuestros propósitos importa solamente que el aprendizaje ocurre en el nivel del sistema en términos del incremento de la inclusión (universalismo), generalización de valores y especificación de metas, pero que es explicado en términos de las propiedades de la acción individual. Habermas se apodera de este modelo, agregándole una perspectiva cognitiva a la teoría de la acción, enfatizando el carácter constructivo del individuo en el proceso de socialización (y este constructivismo se basa en ideas sacadas de la teoría gramática generativa y del cognitivismo piagetiano y transformada en una teoría de la competencia comunicativa - la famosa teoría de la acción comunicativa, la cual sigue siendo básicamente una teoría individualista de la acción). Aquí, nuevamente, la idea es que la evolución de los sistemas sociales puede ser explicada por una "teoría de la acción", lo que significa, en referencia a la orientación de las acciones de actores individuales.

En Habermas esto resulta, en última instancia, en el intento por situar en paralelo el aprendizaje individual y evolutivo en un doble sentido (Habermas 1979). El primero es que el conocimiento acerca del desarrollo humano revelará la capacidad posiblemente universal de los humanos de desarrollar sus potencialidades otorgadas por el lenguaje; tal potencial podría ser identificado reinterpretando las teorías psicológicas piagetianas acerca del desarrollo cognitivo, en teorías de aprendizaje individual con un fin incorporado, a saber, la reflexividad cognitiva y la argumentación moral universal. Teniendo así un modelo del potencial desarrollo humano individual, los macro-desarrollos podrían ser entendidos como procesos de aprendizaje evolutivo, al postular una interacción entre el aprendizaje individual y los desarrollos socioculturales. La evolución cultural - se concluye - es dependiente de las capacidades individuales de aprendizaje y el que ocurra la evolución cultural garantiza la reproducción de tales capacidades, las cuales en cambio aumentan el nivel de los posibles procesos de aprendizaje individuales. De esta manera, una teoría interactiva de los desarrollos a nivel individual y sistémico es la base para una teoría de los procesos evolutivos.

Esta teoría aún le debe mucho a la Ilustración temprana, la cual fue poco clara acerca de su propia función en la emergencia del mundo moderno. Parsons y Habermas parecen nuevos herderianos, quienes aún piensan en términos de individuos que han de ser educados, agregando la idea de una reacción constructiva por parte del educado, y debido a esto, argumentando por cualquier acto simbólico que pudiera ayudar a las personas a aprender algo. El mejor ejemplo de estas implicaciones prácticas es la propuesta de Habermas, de que para aprender un nuevo paso hacia la cultura democrática, los alemanes debieran dedicarse a una discusión constitucional, siendo esta discusión vista como un medio para aprender. Aquí es obvio el modelo de un intelectual lanzado a una discusión con la intención de hacer aprender a las personas. Esta proposición tiene cierto parecido a lo que los alemanes llaman 
"Oberlehrerverhalten" (comportarse como un profesor de educación secundaria). Esta proposición habermasiana no ha sido asumida (en sí mismo, un evento sociológicamente interesante). Asumo que este es el caso, no porque los alemanes son malos demócratas que no recibieron suficientes clases sobre democracia, sino simplemente por el hecho de que el aprendizaje de las sociedades no ocurre de esta manera ${ }^{10}$.

El modelo de socialización va en contra de la lógica de aprendizaje en sociedades realmente modernas. La modernidad ha excluido el modelo educacional de aprendizaje porque es demasiado cercano a un modelo jerárquico, el cual contradice las premisas básicas de la modernidad ${ }^{11}$. Así, la opción teórica para entender el aprendizaje evolutivo en términos del modelo educacional no es viable. ¿Cómo se ve entonces un modelo viable apropiado para las reales sociedades modernas? ¿Y cuáles son las implicancias en relación a las implicancias normativas del acercamiento habermasiano, el cual representa una importante línea del pensamiento social moderno acerca del desarrollo y aprendizaje social?

De esta manera, el enfoque de la teoría de la socialización sobre el aprendizaje evolutivo debe ser corregido en dos aspectos diferentes. Primero, debe ser corregido en lo que respecta sus supuestos individualistas, los cuales dicen que el agregado de personas educadas va a aprender. Segundo, debe ser corregido respecto al modelo relacional implícito, el cual aún se encuentra en deuda con las características de la tradición jerárquica para las culturas de la temprana modernidad y las culturas universalistas no modernas. Una sociología de los intelectuales no es ya la clave del aprendizaje social ${ }^{12}$.

La alternativa es abandonar la idea del aprendizaje individual y generalizar la idea de algunos enseñando a otros qué aprenden ${ }^{13}$.

La base micro-sociológica de los procesos de aprendizaje evolutivos ha de ser conceptualizada no en términos de una teoría de la acción social, sino en términos de una teoría de la interacción social; no en términos de sujetos competentes, sino en términos de formas evolutivas de intersubjetividad; no en términos de intenciones, sino en términos de relaciones. Lo que esto significa exactamente será clarificado a continuación.

\footnotetext{
${ }^{10}$ Esto también explica la centralidad de la sociología de los intelectuales para explicar la evolución cultural en las sociedades modernas (y hasta de las sociedades premodernas con religiones universalistas).Ver entre muchos otros, la discusión de Eisenstadt acerca del rol de los intelectuales en la historia (Eisenstadt 1981, 1982).

${ }^{11}$ Puedes educar a los jóvenes, pero ya no a los adultos.

${ }^{12}$ Tampoco la sociología de la vida cotidiana nos dice algo acerca del aprendizaje. Sin embargo, el interaccionismo simbólico, especialmente el trabajo de Goffman, nos ayuda a corregir para una perspectiva relacional a la cual debemos agregarle el tema de cómo el orden, los marcos, etc. son cambiados en el curso del cambio social o de la evolución social.

${ }^{13}$ Ver Strydom $(1992,1993)$ para una crítica sensible de Habermas y su potencial.
} 


\subsection{Una opción teórica alternativa: interaccionismo}

Las tradiciones teóricas que parten del individuo ${ }^{14}$ permanecen atascadas en sus supuestos individualistas y son solamente consecuentes cuando finalmente declaran que su programa de investigación es básicamente individualista, ya sea por supuestos acerca de las capacidades cognitivas humanas o por supuestos acerca de sus motivaciones racionales. Una teoría no psicológica (y no individualista) de la acción se basa en la idea de que la base del aprendizaje social no son los individuos, sino las relaciones sociales. Así, no es importante lo que las personas tienen en mente, sino lo que comparten entre $s^{115}$. Hasta el modelo educacional clásico supone una relación social: una relación jerárquica que le permite a uno ir desde arriba hacia abajo, desde el educador hacia el individuo que aprende. Así, las relaciones jerárquicas pueden ser explicadas como una forma especial de relaciones sociales, por ejemplo como una relación basada en el poder. Al mismo tiempo, una teoría relacional no usa ya al individuo como una unidad básica de análisis, sino las relaciones entre ellos. Para una teoría del aprendizaje, esto ofrece la posibilidad de ver el modelo educacional en términos relacionales y conceptualizar las teorías del aprendizaje a lo largo del continuo de modos jerárquicos e igualitarios de aprendizaje $\mathrm{e}^{16}$.

Tal desplazamiento no es el resultado de una lógica inmanente de pensamiento sobre el aprendizaje (si es que existe tal cosa como una lógica paradigmática), sino que tiene que ver con reales cambios en la manera en que el aprendizaje social ha sido observado por aquellos involucrados en procesos de aprendizaje colectivos. El punto central es que la cultura moderna es percibida por sus participantes como el resultado de una empresa colectiva, como el resultado de una discusión colectiva, de argumentación colectiva en grupos, organizaciones, instituciones. ${ }^{17} \mathrm{La}$ producción de la cultura moderna es percibida por los actores involucrados como un proceso de aprendizaje colectivo (principalmente de una clase, a saber, el 'Bildungsbürgertum'). De esta manera, el entendimiento de la sociedad moderna en sí misma nos fuerza a avanzar desde una teoría individualista a una teoría relacional de la acción social.

Este punto ha sido dejado en claro - con nuevos costos asociados al entendimiento de los procesos de aprendizaje evolutivos - por Luhmann ${ }^{18}$. Su énfasis en una perspectiva relacional de la sociedad y su reconstrucción de los procesos auto-or-

\footnotetext{
${ }^{14}$ Esto se mantiene no solamente para la arriba mencionada tradición de Parsons/Habermas, sino también para las teorías de elección racional, las cuales se basan igualmente en supuestos individualistas. Las últimas difieren simplemente al asumir un modelo más simple del aprendizaje y el comportamiento individual. Para una discusión de esto, ver Eder/Schmidtke (1998).

${ }^{15} \mathrm{Si}$ lo que comparten, es más o menos lo que tienen en mente, es un punto importante para futuras discusiones sobre los procesos de aprendizaje social.

${ }^{16}$ Los argumentos para una perspectiva relacional han sido recientemente resumidos por Emirbayer (1997).

${ }^{17}$ Esto ha sido enfatizado por Habermas en su libro sobre la idea del espacio publico ("Öffentlichkeit").Ver tambien en Eder (1985) el extenso trato sobre las formas históricas a través de las cuales esta idea de situaciones de interacción igualitaria y discursiva ha evolucionado.

${ }^{18}$ Para una introducción general sobre la teoría de la sociedad como un sistema auto-organizado, ver Luhmann (1995).
} 
ganizativos y las capacidades auto-organizativas de los sistemas sociales indican el cambio necesario en la perspectiva. El aprendizaje es algo que ocurre en el proceso de interacción, y su representación en una mente individual es simplemente un (y frecuentemente el menos importante) factor que explica cambio o procesos de aprendizaje evolutivo ${ }^{19}$. Lo que se pierde en la teorización de Luhmann es la idea de un mecanismo micro social de aprendizaje. Aunque toma una perspectiva adecuada, le quita a la realidad social cualquier supuesto sobre lo que está ocurriendo en los micro-fundamentos de los macro-procesos. La teoría no individualista es como la diva desnuda (o rey) que ha de comprar nueva ropa.

\subsection{Dos modelos de interaccionismo}

Hay dos maneras de darle nueva ropa a la diva (o al rey). Una opción es llenar la teoría interaccionista con supuestos racionalistas acerca del carácter relacional de la realidad social. La otra es llenarla nuevamente con supuestos que están contenidos en la teoría individualista de la acción habermasiana. Solamente daré dos indicaciones de cómo se podrían ver estos rellenos de la teoría. Respecto a la redefinición racionalista del modelo relacional, el aprendizaje evolutivo puede ser entendido en varias maneras. La más sencilla sería formular una teoría del cambio en la estructura de las preferencias, como el resultado de una serie de situaciones de elección racional. El modelo de juegos iterativos de Axelrod provee un modelo más bien limitado de tal proceso de aprendizaje: es la explicación de tan sólo un posible proceso de aprendizaje al interior del modelo (Axelrod 1984, 1986). Otra manera sería concebir el aprendizaje como el efecto de una cooperación racional, como acuerdos colectivos sobre reglas del juego, de manera de coordinar de antemano la acción individual, motivados por la evasión de posibles resultados irracionales (Elster 1989). Una solución aun más radical para el aprendizaje dentro de este paradigma sería argumentar que la acción racionalmente motivada nos fuerza a crear acuerdos normativos, de manera de evitar resultados irracionales. Este argumento se basa en la observación de que ser racional no excluye que es racional ser irracional. Siendo este el caso, las normas deben de ser definidas y acordadas de manera de generar un mínimo de racionalidad social, para poder continuar existiendo dentro de las relaciones sociales desde las cuales las acciones comienzan. La interesante implicancia de esto es que la racionalidad no se encuentra ya ubicada en el individuo, sino en el o los contextos sociales a los que se encuentra vinculada.

El aprendizaje de las sociedades, entonces, es un fenómeno que no puede ser explicado por medio del aprendizaje individual, dado que es un efecto de la coordinación social de procesos de aprendizaje de individuos. La coordinación social, sin embargo,

\footnotetext{
${ }^{19}$ La teoría del carisma es un buen ejemplo.
} 
sigue una lógica distinta a la lógica del aprendizaje individual. Esta teoría puede informar a una teoría del aprendizaje social en el grado que los procesos de aprendizaje puedan ser explicados como intentos de entorpecer la acción irracional ${ }^{20}$. El mecanismo descrito es un mecanismo básico de aprendizaje social, desde el aprendizaje grupal interpersonal hasta el aprendizaje evolutivo. Se podrían dar ejemplos en referencia a la emergencia del Estado, de normas dietéticas y de normas contractuales, hasta la emergencia de normas contractuales en la negociación, etc. ${ }^{21}$

La manera alternativa de rellenar el modelo relacional es volviendo a algunos de los supuestos sustanciales de la teoría habermasiana, a saber, que los individuos basan su capacidad de actuar racionalmente en competencias cognitivas y morales específicas (i"no-ser-capaz-de-no aprender" - ver Habermas 1979:147 - fue la manera inicial de justificar este tipo de aprendizaje! $)^{22}$. El argumento de un semejante enfoque de teoría de la comunicación funcionaria así: De manera de desarrollar tales competencias, ciertas situaciones micro sociales son necesarias. Esto nos daría una clave para distinguir entre situaciones que contribuyen al aprendizaje de los individuos y aquellas que no.

Un buen ejemplo es el modelo de procesos de aprendizaje colectivo que propone Miller (1986). Él define el proceso de aprendizaje colectivo "ideal" como el intento de solucionar inconsistencias cognitivas a través de una argumentación racional que requiere que los principios de generalización, de objetividad y de verdad sean seguidos en tales situaciones argumentativas. Esta situación ideal sirve así a la identificación de situaciones en las cuales el aprendizaje es o bien evitado, interrumpido o sistemáticamente restringido, lo que desemboca en una tipología de procesos de aprendizaje bloqueados, lo que él denomina como aprendizaje autoritario, ideológico y regresivo. El que el aprendizaje sea evitado no se debe al hecho de que los individuos no aprendan, sino al hecho de que se encuentran involucrados en procesos de aprendizaje colectivos, en los cuales las relaciones entre individuos "neutralizan" los requisitos para la argumentación racional.

Un primer tipo de bloqueo de procesos de aprendizaje colectivo se da en situaciones cuando el conjunto de conocimientos es aceptado por su mera autoridad. El principio de que cualquier postulado debiera estar sujeto a una prueba de su generalizabilidad ya no es aplicado. Esto es aprendizaje autoritario. ${ }^{23} \mathrm{El}$ aprendizaje ideológico es más sutil. Principalmente dice que en ciertas áreas, tales como las

\footnotetext{
${ }^{20}$ Esto se podría convertir, si no se trata con suficiente cuidado, en una teoría más bien reaccionaria, no muy distinta de la idea de Gehlen acerca de las instituciones que sirven de baluartes de la racionalidad contra la potencial irracionalidad de la acción individual.

${ }^{21}$ Existe amplia literatura respecto a este argumento. Sobre la emergencia del Estado, ver trabajos tales como Claessen/ Skalnik (1978), Harris $(1977,1978)$. Respecto a los regímenes dietéticos, el debate entre Harris y Sahlins es informativo (Harris 1979; Sahlins 1976). Para el problema de las normas contractuales, ver Elster (1991).

${ }^{22}$ Ver como una aplicación al problema de la emergencia del Estado en Eder (1976).

${ }^{23} \mathrm{El}$ análisis sobre pensamiento autoritario de Adorno (Adorno et al. 1969 [1950]) encuentra aquí una explicación sistemática.
} 
preguntas morales o normativas, ya sabemos cuáles son las respuestas correctas. Esto implica que el principio de objetividad puede ser suprimido, el cual supone que los argumentos del otro (el enemigo) no son vistos como parte de la definición de la situación entre los que participan en el proceso argumentativo, hasta cuando no hay argumentos en contra de esta postura. Sistemáticamente se excluyen argumentos del proceso argumentativo. El aprendizaje regresivo es el caso más radical y claro de procesos de aprendizaje bloqueados, porque en estos procesos de aprendizaje la idea de racionalidad argumentativa, el principio de verdad, es dejada de lado. Esta es la condición de la irracionalidad colectiva, donde cualquier intento por coordinar la propia acción con la acción de otros lleva a ansiedad, a retirarse de formas de argumentación racional. No es difícil imaginar tales situaciones, desde situaciones de doble atadura (es decir, casos psiquiátricos) hasta el discurso stalinista.

Este enfoque de teoría comunicativa sobre el aprendizaje, donde la referencia básica no son ya los individuos, sino las relaciones entre ellos, nos lleva a la idea de que la racionalidad de los discursos se encuentra atada a la organización social de estos discursos. Así, la idea de racionalidad cambia desde una base en el individuo, a la situación social en la cual actúa ${ }^{24}$. Pero hay un problema involucrado en tales supuestos racionalistas. Puede ser cuestionado si hay algo más fundamental a la situación relacionalmente definida de actores interactuando que la racionalidad, algo que va más allá de las presunciones de racionalidad de sujetos que interactúan. ¿El supuesto de racionalidad le sigue debiendo mucho a la idea de acción racional?

\subsection{Los cimientos narrativos del orden social}

Seguimos viviendo demasiado a la sombra de Max Weber y su idea de que para entender lo social debemos entender el significado que los actores le ponen a sus acciones. El problema de cómo llegar desde los significados subjetivos hacia un orden social ha sido resuelto por Weber - como sabemos - construyendo tipos ideales de acción. Es el supuesto de que existe una cierta racionalidad para cada acción lo que nos permite entender la acción social de otros, simplemente por el hecho de compartir con ellos tales estándares de racionalidad. Es obvio que esta ya es una inter-

\footnotetext{
${ }^{24}$ Existe un movimiento similar dentro de la teoría de la elección racional. Las diferencias entre un enfoque de elección racional y un enfoque de teoría de la comunicación (o cognitivo), sin embargo, no debieran ser minimizadas. Estas son dos nociones fundamentalmente opuestas de racionalidad. La primera tiene que ver con una racionalidad que coordina la acción social a través de cierta forma de mecanismo de mercado. En el segundo caso, tenemos una noción de racionalidad que tiene mucho más que ver con la tradición en la que vivimos, es decir, la modernidad. De esta manera, las diferencias tienen que ver con una relación distinta entre la manera en que pensamos y el mundo en el que hemos aprendido a pensar de esta manera. Supongo que un enfoque cognitivo funciona mejor que un enfoque racionalista - pero este es un tema controversial, a ser seguido en otra discusión. La reflexividad moderna es un fenómeno que puede ser analizado de manera mucho mas adecuada por la última teoría - ¿cómo puede el discurso sobre la necesidad normativa ser analizado más que en referencia a la racionalidad que está incorporada en la misma situación social, a saber, la coordinación racional de argumentos?
} 
pretación de Weber, pero una que considera gran parte de los subsecuentes debates teóricos desde Parsons hasta Habermas e incluso más allá.

Este supuesto ha sido severamente criticado en los recientes años. El criterio de racionalidad es demasiado limitado para ayudar a entender la acción social en términos de su rol en la producción y reproducción del orden social. Especialmente no nos ayuda a explicar qué es la comprensión compartida de una situación en la cual los actores aprenden y contribuyen al conocimiento compartido del mundo. Esta es la crítica del "intelectualismo" en la teorización social (Heiskala 1997). Otra crítica es que tal idea no nos permite entender por qué tanto aprendizaje que sería posible, no ocurre. Parece ser que las sociedades aprenden sólo bajo ciertas condiciones específicas, a saber, cuando una incertidumbre básica permea a la sociedad y destruye el ordenamiento básico de las experiencias con el mundo natural, social y psíquico. Esta es la crítica del "idealismo".

¿Cómo debemos concebir una base no intelectualista y no idealista para construir una teoría sociológica del aprendizaje? Las respuestas clásicas no son suficientes: Volver al sentido común es una estrategia que sigue siendo intelectualista porque reemplaza el conocimiento científico con el sentido común, sin dejar de lado el foco en el conocimiento ${ }^{25}$ : volver al realismo es una estrategia que sigue siendo idealista porque solamente especifica condiciones materiales bajo las cuales las ideas se comprenden a sí misma, sin dejar de lado el foco en las fuerzas causales de las ideas o los significados ${ }^{26}$. Una tercera opción es ir más allá del significado, ir más allá del supuesto de un significado sustantivo de la acción y moverse hacia la manera en la cual se le otorga significado a la acción en el curso de la acción y de la interacción. Este cambio "dramatúrgico" desde el significado hacia el otorgarle significado a algo (Wuthnow, 1987) nos fuerza a observar el proceso a través del cual algo significativo es generado, más que al significado, como tal.

¿Existe una base pre-cognitiva para el entendimiento y la explicación de los procesos sociales de la construcción de significado? La reciente teorización acerca de la base del orden social se orienta hacia preguntas sobre el reconocimiento recíproco de los actores en situaciones sociales (Honneth 1992; Taylor 1992). Lo que vincula una acción a otra acción es su reconocimiento como acción significativa por todos los involucrados. Lo que los actores producen en tales procesos no es un consenso racionalmente sustentado, sino más bien un sentido de identificación colectiva de aquellos que se reconocen entre sí. Las construcciones de identidad involucran darle significado a las actividades que no reclaman una racionalidad en particular; son actividades que producen un orden social mas allá o anterior a un orden normativamente justificado. Con el propósito de simplificar, podemos llamar a tal orden, un

\footnotetext{
${ }^{25} \mathrm{La}$ tradición fenomenológica es tan cognitivista como su contraparte weberiana. Difiere solamente en sus premisas metodológicas respecto a la relación entre el sentido común y el conocimiento científico.

${ }^{26}$ Esta tradición realista es especialmente pronunciada en el trabajo reciente de John Searle (1995). Una buena discusión al respecto se puede encontrar en Corsten (1997).
} 
orden narrativo. En las explicaciones narrativas, los actores se reconocen a sí mismos y a los otros, y sin embargo, se encuentran vinculados a un mundo de reconocimiento recíproco.

Tales órdenes narrativos pueden hasta incluir tipos de acción y conocimientos racionales. La acción racional (en el sentido de Weber), se convierte en significativa en el orden narrativo de una interacción social. La racionalidad es entonces sólo un caso especial de una lógica de acción más abarcadora. Un caso instructivo es la nueva teoría institucional. Ella argumenta que las instituciones se basan en órdenes narrativos, no en racionalidades. Esta idea (Powell/diMaggio 1991) provee otro caso para la reorientación del pensamiento social, lejos de las ilusiones de la acción racional. Lo que las instituciones hacen y piensan no es más que usar modelos racionales de acción para racionalizar lo que ellas están haciendo, para dar una explicación narrativa de su racionalidad. La tesis que proponen teorizaciones más recientes es que las acciones no se relacionan entre sí por cierto estándar de racionalidad implícita, sino que por cierto orden narrativo que le otorga significado a la propia acción en relación a la acción de otros. De esta manera, no debemos seguir luchando con la diferencia en la calidad de la racionalidad. Pero debemos comprender lo que se encuentra detrás de la racionalidad. Wuthnow ha llamado a esta estrategia "teoría dramatúrgica", porque ésta enfatiza los procesos a través de los cuales los significados (racionales, tanto como irracionales o significados a-racionales de la acción) se hacen significativos ${ }^{27}$. El objetivo de ir "mas allá del significado" se vincula a una base trans-subjetiva de la acción e interacción social. La afirmación es que la organización narrativa de la experiencia provee las características estructurales de las situaciones sociales en las cuales los participantes en tales situaciones, ya sea tienen opciones de aprender o son forzados a no aprender. ${ }^{28}$

Esta discusión hasta ahora ha dado una respuesta a la pregunta de cómo ocurre el aprendizaje. Ocurre en situaciones en las cuales los procesos de interacción producen efectos que modifican el conocimiento colectivamente compartido. La respuesta más general a la pregunta por el "cómo" es que en las situaciones sociales se genera algo "nuevo". Cuando tal aprendizaje ocurre, produce resultados específicos: El aprendizaje cambia a) los marcos y creencias normativas básicas que guían la acción social o b) el conocimiento empírico acerca del mundo, utilizado como un recurso en la acción social.

Hasta ahora la pregunta acerca del aprendizaje ha sido reformulada al optar por un acercamiento interaccionista. Ya no nos preocupamos del aprendizaje de niños

\footnotetext{
${ }^{27} \mathrm{El}$ fundador de tal perspectiva es Goffman, quien mostró especialmente en el "frame analysis" como las personas representan a través de reglas específicas el significado de cualquier acto o evento, es decir, le otorgan significado a algo a través de la acción. Al respecto ver también Swidler (1986).

${ }^{28} \mathrm{La}$ estructura narrativa de los órdenes sociales describe una tendencia de reciente importancia en la teorización social. Ver por ejemplo, Somers (1994). Mientras los sociólogos más tratan con y descubren formas de conocimiento que se alejan del conocimiento cognitivo modelado de acuerdo al estándar de la ciencia, más se dirigen hacia teorías y metodologías narrativas.
} 
que son criados para alcanzar su mayor potencial. Más bien, tenemos adultos que interactúan, que aprenden en el curso de su interacción, en situaciones en las cuales cierta narrativa básica provee el terreno básico para la comprensión y comunicación recíproca. Pero, ¿qué aprenden? Esta pregunta nos lleva a una segunda problemática, otra forma de salirse del modelo clásico del aprendizaje social. Esto tiene que ver con la pregunta respecto a qué aprenden las sociedades cuando aprenden. La pregunta ahora es qué es aprendido en situaciones narrativamente ordenadas que proveen de estructura a las relaciones sociales. Así, la institucionalización del conocimiento debe ser observada: es decir, la producción de conocimiento y de formas sociales que recolectan y retienen tal conocimiento más allá de la capacidad de la memoria individual.

\section{3. ¿Qué aprenden las sociedades?}

\subsection{Conocimiento sustantivo y reglas de procedimiento}

Cuando las sociedades aprenden, no se producen a sí mismas, sino que producen cultura. Esto puede parecer obvio. Sin embargo, el debate teórico de los últimos años, especialmente en el despertar de los estudios culturales, ha dejado borrosa esta diferencia central. Cultura y sociedad son dos cosas distintas. Su amalgamación tiene tradiciones venerables, especialmente en la antropología social y cultural, cuando los etnógrafos definen a la sociedad que estudian como una cultura ${ }^{29}$. Ellos amalgamaron lo que una sociedad produce como una representación de sí misma, con la sociedad. De manera de clarificar este punto, usaré la distinción entre aprendizaje sustantivo y aprendizaje de reglas.

Cuando las sociedades aprenden, producen un stock de conocimiento común a través del cual construyen una realidad social. Para hacer esto, ellas crean reglas cognitivas compartidas para clasificar e interpretar la información que fluye alrededor de la sociedad. Los procesos de aprendizaje que llevan a la acumulación de conocimiento serán definidos como "aprendizaje sustantivo". Esto desemboca en la idea de una cultura de la sociedad, la cual está hecha de diferentes tipos de conocimientos, desde el sentido común hasta el conocimiento científico. En vez de comprender el aprendizaje social como un proceso en fases, que va desde un sentido común primitivo a un conocimiento científico racional, propongo la idea de un proceso multidimensional de aprendizaje acumulativo, que ocurre en todos los niveles del conocimiento simultáneamente. El conocimiento acumulado, sin embargo, es solamente significativo cuando puede ser usado por los actores sociales, cuando está ordenado de una manera que permite la comunicación del conocimiento en una sociedad.

\footnotetext{
${ }^{29} \mathrm{La}$ separación de sociedad y cultura en dos entidades distintas es un argumento central en Archer (1988).
} 
Las sociedades también aprenden algo más. Aprenden a crear situaciones en las cuales la experiencia puede ser almacenada y traspasada de generación en generación. Aprenden a estabilizar la información que ha sido generada o que está siendo generada en una sociedad. Las sociedades aprenden cómo aprender. Ellas aprenden procedimientos de cómo generar nuevo conocimiento y de cómo asimilar nuevo conocimiento en el stock de conocimiento existente. Este aprendizaje es definido como el aprendizaje social de reglas, en oposición al aprendizaje sustantivo ${ }^{30}$.

\subsection{La producción de conocimiento}

El aprendizaje sustantivo ha sido el foco principal de las teorías de la evolución cultural. Podemos reconstruir la acumulación de conocimiento tecnológico, así como la racionalización del orden moral hacia el universalismo (y su especificación hacia universalismos en competencia). La teoría de Piaget acerca del desarrollo cognitivo ha sido usada para reconstruir procesos paralelos a nivel histórico (el mismo Piaget presentó una enorme explicación de tres volúmenes acerca de la evolución del conocimiento cognitivo en la historia). Habermas, continuando esta línea de pensamiento, y vinculándola a la idea de Weber acerca de la racionalización como el proceso principal en la evolución cultural, ha agregado la idea de un proceso de racionalización moral a lo largo de la historia (Habermas, 1987).

Dado que nadie en este mundo puede abarcar la pregunta acerca de qué ha sido aprendido en la historia, se han hecho simplificaciones, las cuales han provocado crítica inmediata por parte de historiadores de la ciencia, la tecnología, la religión, y por parte de todos los especialistas de estudios regionales, tales como los orientalistas, africanistas, etc. Una manera de simplificar esta tarea ha sido reconstruir los supuestos básicos de este conocimiento. Una línea terminó en las filosofias de la ciencia, lo que reveló el movimiento ideal de las ideas detrás del movimiento real de las ideas, una estrategia que pronto perdió credibilidad. Otra línea ha sido reconstruir estructuras paradigmáticas y mostrar su estructura temporal (esto es el estructuralismo genético, en el sentido de Piaget o Habermas). La tercera alternativa ha sido la "crítica": criticar los arreglos cognitivos a través de los cuales las culturas se describieron mutuamente, un juego cognitivo que puede ser romántico (no entendemos a los primitivos) o colonialista (los primitivos no nos entienden a nosotros) o deconstructivista (nadie puede entender al otro). ${ }^{31}$

El aprendizaje, en el sentido del conocimiento acumulativo, ciertamente ha ocurrido en la historia. Sin embargo, tal proceso acumulativo se debe simplemente al tiempo.

\footnotetext{
${ }^{30} \mathrm{La}$ acumulación es ciertamente también un aprendizaje basado en reglas. Así, la diferencia entre estos dos tipos de aprendizaje es en menor medida su carácter de regla, que sus diferentes tipos de reglas involucradas: las primeras series de reglas son reglas de ordenamiento cognitivo, las segundas series de reglas son reglas de procedimientos para organizar el mundo social.

${ }^{31}$ En este contexto, la pregunta acerca de la comprensión de otras culturas ha ganado ímpetu.
} 
Mientras las personas tienen más experiencias, más acumulan (y olvidan) tales experiencias..$^{32}$ Parece ser que tal pregunta teórica está simplemente mal planteada. La respuesta es la obvia o no existe respuesta alguna. Pasemos por lo tanto al aprendizaje social de reglas, el cual lleva la pregunta hacia el problema del aprendizaje "estructural", del aprendizaje de nuevas estructuras para generar y reproducir conocimiento.

\subsection{La producción de reglas para el aprendizaje: el aprendiza- je del aprendizaje}

El aprendizaje de reglas es diferente al aprendizaje sustantivo. El conocimiento siempre se relaciona a alguna manera de organizar la experiencia. El aprendizaje de reglas es un aprendizaje social, en el sentido de que el conocimiento se organiza y reproduce en una forma de vida social, la que define un set específico de reglas para organizar y almacenar conocimiento. Tal perspectiva basada en las reglas, nos aleja del "modelo educacional", el cual argumenta que debemos identificar al educador, de manera de explicar porqué los actores sociales aprenden. Esto nos lleva más bien a un modelo de procesos de aprendizaje auto-organizativos, los cuales están organizados como procesos comunicativos estructurados por reglas. Las reglas de la comunicación de conocimiento se convierten así en la clave del aprendizaje social.

El aprendizaje de reglas puede ser analizado distinguiendo situaciones comunicativas que son construidas en el proceso de aprendizaje.Voy a distinguir tres niveles de aumento de inclusividad, cada uno teniendo sus propiedades específicas. Estos tres niveles son el nivel interpersonal, el nivel organizacional y el nivel institucional. Estos niveles serán discutidos en este mismo orden.

El aprendizaje interpersonal se refiere a situaciones donde el aprendizaje ocurre a través de una comunicación directa. Esto aplica a cualquier situación, ya sean niños aprendiendo colectivamente cómo juzgar un fenómeno empírico o cómo solucionar conflictos morales, ya sea la interacción directa entre científicos tratando de resolver evidencia contradictoria y replanteando sus códigos éticos o hacia la acción política. Un primer ejemplo es el estudio de asociaciones, concebidas como redes interpersonales creadas con propósitos políticos, culturales o sociales. Personalmente traté con este problema en el nivel de asociaciones políticas emergiendo con el ascenso de la sociedad civil moderna en Alemania en el siglo XIX (Eder, 1985). Estas sociedades de la Ilustración (“Aufklärungsgesellschaften") no solo generaron un marco o paradigma conceptual particular para interpretar sus experiencias políticas, sino que también aprendieron que estaban aprendiendo colectivamente. Aprendieron reglas a través de las cuales generar, estabilizar y eventualmente modificar sus

\footnotetext{
${ }^{32}$ Obviamente, el conocimiento ha aumentado en las sociedades modernas. Sin embargo, la teoría evolutiva es demasiado general, si no fuera posible decir más que eso. Este es el límite de la teoría evolutiva clásica para las ciencias sociales. Para este argumento las contribuciones ver la obra de Schmid/Wuketits (1987).
} 
paradigmas o marcos conceptuales. La lógica que emergió (luego de algunas décadas de ambivalencia relacionadas al modelo educacional, por ejemplo, en grupos masones) puede ser encontrada en la idea de una situación estructurada de acuerdo a los principios de igualdad de los miembros y de discursividad de las interacciones. Esto se acerca al modelo discursivo que Habermas identificó como prácticas racionales subyacentes de actores sociales bien socializados. Wuthnow los ha llamado "comunidades de discurso" (Wuthnow, 1990). Haas los llama "comunidades epistémicas" (Haas, 1992).

Los patrones de interacción científicos y la manera en la cual las reglas de interacción han sido inventadas y establecidas en comunidades científicas han sido estudiados en el trabajo micro-sociológico de Knorr-Cetina (1981). Un ejemplo histórico son las sociedades científicas (siendo la Royal Society una de las mejor estudiadas), las cuales reorganizaban sus relaciones interpersonales de acuerdo al modelo de membrecía libre e igualitario de tales sociedades. No sólo inventaron nuevo conocimiento (esto ha sido secundario), sino que practicaron procedimientos para producir, revisar y publicar nuevo conocimiento acerca del mundo. Inventaron reglas para el trabajo inventivo. Estos grupos son "comunidades epistémicas", una etiqueta de moda en sociología del conocimiento y de la ciencia en años recientes (Haas 1992).

El aprendizaje organizacional muestra de manera aún más clara el rol de las reglas en el aprendizaje social. Las organizaciones están definidas como diseños para el aprendizaje organizacional, para adquirir información acerca del medio ambiente de la organización y para mejorar lo que la organización puede hacer con este medio ambiente. Para entender tal aprendizaje organizacional, debemos saber cómo se almacena el conocimiento en una organización, cómo es transmitido a una población cambiante, cómo se trata con el cambio en las organizaciones ${ }^{33}$. De esta manera, el aprendizaje organizacional es el producto necesario de cualquier forma organizacional de vida social. El aprendizaje es un proceso de búsqueda y procesamiento de información acerca del medio ambiente, de manera de reducir las incertidumbres con las cuales se enfrenta cualquier organización mientras trata con el medio ambiente. La supervivencia de una organización depende de su capacidad de reducir las incertidumbres de su medio ambiente a través del aprendizaje (Simon 1991).

El que el aprendizaje sea mejor que la maximización de ventajas (la exploración es mejor que la explotación) ha sido enfatizado en literatura reciente sobre la estabilidad organizacional. Esto significa, en términos del aprendizaje social de reglas, que las organizaciones tienen que construir rutinas que permitan el aprendizaje. El repertorio de rutinas provee reglas para la adquisición, la recolección, el almacena-

\footnotetext{
${ }^{33}$ Ver las contribuciones a la "Ciencia Organizacional" en Febrero de 1991 (Cohen/Sproul, 1991), especialmente la contribución hecha por March, Cohen, Simon y Levinthal. La contribución hecha por March (1991) se vincula a la necesidad de entender las dinámicas de la lógica de la toma de decisiones (March 1988). Una visión general de la discusión sobre el tema se encuentra en Levitt/March (1988). Formulaciones previas de este problema pueden ser encontradas ya en Argyris/Schön (1978).
} 
miento y la transmisión de información en una organización. De manera simultánea, las relaciones entre aquellos que realizan tal adquisición, almacenamiento y transmisión deben ser ordenadas por cierta regla de comunicación organizativa al interior de las organizaciones, de manera de definir las premisas de decisión, para enmarcar los problemas organizacionales, etc. Las estructuras organizacionales son en sí mismas un set de reglas para tratar con este problema ${ }^{34}$.

El aprendizaje institucional se refiere a las reglas que coordinan a los actores organizacionales. Las instituciones aprenden al definir espacios inter-organizacionales y poniéndole nombres a estos espacios. Así, las instituciones permiten que hayan comunicaciones entre actores organizacionales y restringen, al mismo tiempo, el modo de comunicación por medio de reglas normativas y cognitivas, proveyendo un orden moral con sustento institucional y un espacio cognitivo, dentro del cual se organizan las actividades comunicativas.

El nivel más abarcador de los órdenes institucionales es el espacio público dentro del cual ocurren los discursos. Nos referimos aquí al discurso, en su doble sentido, de universos discursivos y de prácticas discursivas (o procedimientos). Así definido, el aprendizaje institucional es o bien el resultado, o bien el proceso del discurso público. El resultado es la experiencia institucionalizada de una sociedad. El proceso es la puesta en escena de discursos para comunicar experiencia institucionalizada ${ }^{35}$.

Las sociedades modernas se encuentran particularmente formadas por reglas y prácticas discursivas. Las instituciones modernas se encuentran vinculadas al consenso público, como base de su legitimidad. El aprendizaje institucional es requerido por la presión por proveer evidencia de cumplimiento de tales expectativas. Creadores de mitos, intelectuales y periodistas, todos contribuyen al aprendizaje institucional al proveer una reconstrucción de la experiencia institucionalizada. Al hacer esto, también definen las reglas a través de las cuales tal conocimiento colectivo puede ser transformado. En el proceso de reconstrucción de la experiencia institucionalizada, ellos generan un sistema de reglas basado en la libertad de expresión y en el debate crítico. Este ejemplo de aprendizaje institucional es característico de un tipo específico de sociedades modernas. Otros sistemas institucionales en las sociedades modernas generan otras formas de aprendizaje institucional ${ }^{36}$. El punto teórico es que podemos identificar el aprendizaje de reglas ocurriendo en el proceso de construcción de instituciones para coordinar una pluralidad de actores colectivos.

\footnotetext{
${ }^{34}$ Un caso interesante es la aplicación de esta teoría al aprendizaje de organizaciones de movimientos sociales. Tal aprendizaje es necesario para crear y reproducir la acción colectiva. El aprendizaje organizacional explica, en parte, las transformaciones de la acción colectiva en los movimientos sociales modernos. Para una aplicación de esta teoría al aprendizaje en el ámbito del medio ambiente, ver Wynne (1992).

${ }^{35} \mathrm{El}$ concepto de discurso es aún muy maleable. Aparece bajo premisas de Foucault y bajo premisas de Habermas. Aquí estamos interesados en su doble estructura: significando un sistema estructurado de conocimiento y una práctica de conocimiento debatido.

${ }^{36}$ Mary Douglas argumentaría que tenemos al menos cuatro tipos: igualitarios, jerárquicos, individualistas y fatalistas. Ver el desarrollo de su trabajo original (Douglas 1966) hacia una teoría desarrollada de la cultura (Thompson et al. 1990).
} 


\section{4. ¿Por qué aprenden las sociedades?}

¿Para qué sirve una teoría del aprendizaje? ¿Por qué debemos preocuparnos con teorías del aprendizaje más allá de las discusiones teóricas clásicas acerca de cómo conceptualizar el mundo social? Esta es la pregunta acerca de la transición desde modelos conceptuales a modelos explicativos. El que los modelos explicativos asuman modelos conceptuales buenos y diferenciados es - al menos para mí - obvio. Por lo tanto, voy a continuar y, de cierta manera, replantear la pregunta inicial. La pregunta no es ya: ¿Cómo aprenden las sociedades? La pregunta es más bien: ¿Por qué aprenden? ¿Por qué reconocen sus sistemas de creencias y su conocimiento?

Una antigua respuesta, asumida también por Habermas (1979) ha sido: porque noaprender es imposible. El supuesto es que los seres humanos son forzados por la naturaleza a aprender. Esta es la versión individualista de la causalidad. Los humanos luchando por la Ilustración y por el conocimiento son vistos como el mecanismo básico del aprendizaje, en donde ellos finalmente ganarán. Esta es la teoría optimista del aprendizaje social, la cual termina siendo una versión secularizada de la filosofia de la historia. Tal respuesta simplemente implora por la pregunta por explicar el aprendizaje social.

Una respuesta más reciente explica el aprendizaje como una reacción a la incertidumbre (Siegenthaler, 1993). En situaciones de incertidumbre, las personas, organizaciones e instituciones deben reorganizar sus reglas. En fases de rutina, el sentido común, el conocimiento organizacional e institucional son suficientes para hacer crear la diferencia entre lo verdadero y lo falso, lo correcto y lo incorrecto, lo bueno y lo malo. En fases de crisis, tales distinciones se vuelven más difíciles. Las reglas cognitivas para hacer esto no pueden ya ser aplicadas sin incertidumbre.

De manera de cambiar esta situación de incertidumbre, las reglas para tratar con ella son cuestionadas. Las creencias acerca de lo que es compartido colectivamente ya no se mantienen. Es necesario un aprendizaje a través de la comunicación. Las elecciones entre reglas ya no son suficientes. Se deben elegir nuevas reglas. Esto requiere del aprendizaje de nuevas reglas y de la producción de nuevas certidumbres, a través de nuevas reglas acerca de la comunicación de evidencia sobre el mundo de alrededor. En las sociedades modernas tales situaciones de incertidumbre se han convertido hasta en normales: la sociedad del riesgo es un concepto que define a las sociedades modernas como sociedades inherentemente inciertas. No es una sorpresa que en medios riesgosos la pregunta acerca del aprendizaje se haya convertido (tal como en la investigación acerca del riesgo medioambiental) en una categoría analítica y política central ${ }^{37}$. Tal situación, sin embargo, no se mantiene para siempre. Es un caso

${ }^{37}$ Existe una extensa literatura sobre la investigación sobre riesgo y el problema relacionado de cómo convivir con riesgos, de cómo contrarrestar los riesgos. Una buena orientación en el muy diverso debate es ofrecida por Sabatier/JenkinsSmith (1993), Milbrath (1989), Beck (1992) y Wynne (1992). 
límite. Normalmente, las personas siguen teniendo alguna base segura sobre la cual actuar racionalmente en el marco de las reglas, siendo así capaces de evitar el aprendizaje y las consecuentes rutinas. Tiene que haber cierta irritación básica para gatillar los procesos de aprendizaje social.

Cuando tal aprendizaje de reglas se pone en movimiento, modifica las formas sociales a través de las cuales el conocimiento es generado y almacenado. En el caso de una revolución, este sistema de reglas es modificado aún más allá. En tales situaciones, el orden narrativo de la sociedad es destruido. Ya no se cree en las narrativas y nadie puede estar seguro acerca de la comprensión básica de los mundos simbólicos mutuos. Cualquier escenificación del conocimiento arriesga ser ridiculizado. De esto se concluye que las sociedades aprenden cuando su orden narrativo ya no provee el contexto necesario para rituales, rutinas y órdenes normativos. El aprendizaje se encuentra entonces en una nueva narrativa en la cual basar el orden social y su cultura.

Tal aprendizaje de reglas afecta el estado del conocimiento. Deslegitima el conocimiento antiguo y genera nuevo conocimiento. La acumulación de conocimiento puede ser organizada sobre la base de sistemas de reglas que ofrecen nuevas posibilidades para agregar nuevas experiencias al conocimiento existente y que ofrecen nuevas posibilidades para abolir el conocimiento obsoleto. El aprendizaje de reglas de la sociedad es, de esta manera, una condición para cambiar el modo de acumulación de conocimiento. Una teoría del aprendizaje social no es, por lo tanto, una teoría de la acumulación del conocimiento, sino más bien una teoría de la organización y reorganización de sistemas de reglas para la acumulación de conocimiento.

\section{Racionalidad, evolución y aprendizaje}

Para resumir, el aprendizaje es una reacción de los seres humanos hacia las incertidumbres respecto a su experiencia institucionalizada. El conocimiento ya no provee respuestas adecuadas para darse cuenta lo que podría ser lo bueno o lo malo, lo correcto o lo incorrecto, lo verdadero o lo falso. La comunicación acerca de lo que es el conocimiento compartido debe ser gatillado en el nivel interpersonal, organizacional y hasta institucional de la vida social.

Lo que ocurre después no es que los individuos simplemente aprenden y proveen nuevos significados y nuevas reglas para seguir adelante, sino más bien que las relaciones sociales han de ser reorganizadas de manera de abrir espacios de comunicación que permitan la creación de nuevas formas de conocimiento o la reconstrucción de formas viejas de conocimiento. En este proceso, las relaciones sociales son en sí mismas redefinidas. Las reglas de las relaciones sociales de comunicación han de ser encontradas de manera (a) de adquirir nuevo conocimiento, (b) de almacenar tal 
conocimiento y (c) de transmitir tal conocimiento, dada la alternancia natural y/o social de los actores en contextos de comunicación. El aprendizaje es tanto crear universos discursivos como ingresar en relaciones discursivas. Enfrentado con la opción teórica de una primacía del aprendizaje sustantivo o de reglas, la afirmación teórica es que el aprendizaje de reglas es metodológicamente anterior al aprendizaje sustantivo. El supuesto teórico que sigue de esto es que las estructuras intersubjetivas o las reglas son constitutivas de las estructuras subjetivas de conocimiento.

Queda aún una pregunta que es parte del título de este artículo. ¿Por qué es tan difícil cambiar el mundo? La respuesta sería: porque a las sociedades no les gusta aprender. Ellas prefieren quedarse con lo que saben y con las reglas que estabilizan lo que saben. De esta manera, solamente las sociedades que producen riesgos son sociedades donde los actores sociales realmente tienen una opción de cambiar el mundo. Mientras más riesgoso sea el medio ambiente de las sociedades, más aprenden y más quieren cambiar el mundo. La sociedad del riesgo en la que vivimos hoy es un medio ambiente conducente al cambio y al aprendizaje. Contrariamente a las malas noticias acerca de la "sociedad del riesgo", esta sociedad es una de aquellas que precisamente gatilla procesos de aprendizaje (Beck, 1992). Sin embargo, esto crea otro problema. Demasiado aprendizaje confunde.Y los efectos del cambio de conocimiento y normas se encuentran más allá del alcance de las intenciones humanas. De esta manera, el aprendizaje en sí mismo se convierte en un riesgo. Así, ¿no sería mejor para la evolución social parar los procesos de aprendizaje? Lévi-Strauss argumentó en "Tristes tropiques" por una sociedad de este tipo. Dado que es tan dificil cambiar el mundo, no debiéramos cambiarlo, sino vincular al aprendizaje a formas cognitivas y sociales.

Desafortunadamente, es demasiado tarde para esto. Así, tenemos que continuar viviendo en una sociedad que aprende y usar nuestras capacidades de aprendizaje para controlar los efectos no intencionados de nuestro aprendizaje. La evolución social se está acelerando debido a que el aprendizaje social aumenta. Pero mientras más continúa la evolución social, el aprendizaje social moldea en menor medida el futuro. Tratamos de cambiar el mundo por medio del aprendizaje cotidiano, del aprendizaje organizacional (por ejemplo, los reformadores de las universidades), por aprendizaje institucional (inventando nuevas instituciones a través de la acción colectiva organizada y los movimientos sociales) y, aún así, la sociedad evoluciona más bien independientemente de los procesos sociales de aprendizaje. Lo que hacemos es producir efectos a través del aprendizaje social. Los efectos, sin embargo, no son determinados por el aprendizaje colectivo, sino por sus consecuencias sistémicas: por evolución.

La ilusión de la alta modernidad ha sido que creímos que la sociedad aprende porque los grupos, organizaciones e instituciones aprenden. Sin embargo, los grupos, las organizaciones o las instituciones (y ni hablar de las personas) también mueren; aún así la sociedad continúa evolucionando. Podemos cambiar los grupos, las orga- 
nizaciones, las instituciones (hasta a las personas), aún así la sociedad parece resistir los intentos por cambiarla. Al menos, la sociedad no "implementa" los procesos de aprendizaje que ocurren en ella. Existe una diferencia entre el aprendizaje grupal, el aprendizaje organizacional y el aprendizaje institucional, por un lado, y la evolución de la sociedad, por el otro. Las sociedades evolucionan, lo que significa que lo que frecuentemente resulta no tiene nada que ver con las intenciones grupales, las metas organizacionales o los objetivos institucionales. Es a través de las constelaciones macro-sociales y macro-históricas que los procesos de aprendizaje en el nivel grupal, organizacional e institucional se traducen en aprendizaje social. Esto equivale a decir que, tanto como nosotros hacemos la sociedad, la sociedad nos hace a nosotros.

En consecuencia, ¿̇ebiéramos abandonar la idea del aprendizaje social? El aprendizaje es una cosa, la evolución es algo distinto. Debemos separarlos para así darle sentido a los procesos de aprendizaje social. Entonces, ¿para qué sirven los procesos de aprendizaje? Sirven para la evolución porque ofrecen más posibilidades. No cambian el mundo, pero proveen los elementos para cambiar el mundo, para que el cambio siga ocurriendo. Los procesos de aprendizaje en el nivel grupal, organizacional e institucional producen variación de manera de producir suficiente variabilidad para la evolución de la sociedad. Los procesos de aprendizaje en el nivel grupal, organizacional e institucional producen variación y por medio de esto dejan disponible suficiente variedad para la evolución de la sociedad. Producen la materia desde la cual la sociedad selecciona su propia reproducción continua. Esto es evolución basada en procesos de aprendizaje social y, en este sentido, la sociedad aprende. 


\section{Bibliografía}

Adorno,T.W., Fraenkel-Brunswick, E., Levinson, D.J., \& Sanford, R. N. (1969 [1950]): The Authoritarian Personality. New York, NY: Norton Library Edition.

Alexander, J. C., Giesen, B., Münch, R., \& Smelser, N. J. (Eds.). (1987): The MicroMacro Link. Berkeley, CA: University of California Press.

Archer, M. S. (1988): Culture and Agency. The Place of Culture in Social Theory. Cambridge: Cambridge University Press.

Argyris, C., \& Schön, D. A. (1978): Organizational Learning: A Theory of Action Perspective. Reading, MA:Addison-Wesley.

Axelrod, R. (1984): The Evolution of Cooperation. New York, NY: Basic Books.

Axelrod, R. (1986): Modelling the evolution of norms. American Political Science Review, 80, 1095-1111.

Bandura, A. (1977): Social Learning Theory. Englewood Cliffs, NJ: Prentice-Hall.

Beck, U. (1992): Modern society as a risk society. En N. Stehr \& Richard V. Ericson (Eds.), The Culture and Power of Knowledge. Inquiries into Contemporary Societies (pp. 199214). Berlin: de Gruyter.

Beck, U. (1992): Risk Society. Towards a New Modernity. London: Sage.

Boyd, R., \& Richerson, P. J. (1985): Culture and the Evolutionary Process. Chicago, IL: University of Chicago Press.

Corsten, M. (1998): Between Constructivism and Realism - Searle's Theory of the Construction of Social Reality. Philosophy of the Social Sciences, 28, 102-121.

Claessen, H.J. M., \& Skalnik, P. (1978):The early state: theories and hypotheses. In H. J. M. Claessen \& P. Skalnik (Eds.), The Early State (pp. 3-29). The Hague/Paris/New York: Mouton.

Cohen, M. D., \& Sproul, L. S. (Eds.). (1991): Organizational Learning: Papers in Honor of (and by) James March (Organization Science,Vol.2).

Douglas, M. (1966): Purity and Danger. An Analysis of Concepts of Pollution and Taboo. London: Routledge \& Kegan.

Eder, K. (1976): Die Entstehung staatlich organisierter Gesellschaften. Ein Beitrag zu einer Theorie sozialer Evolution. Frankfurt: Suhrkamp. 
Eder, K. (1985): Geschichte als Lernprozeß? Zur Pathogenese politischer Modernität in Deutschland. Frankfurt: Suhrkamp.

Eder, K., \& Schmidtke, O. (1998): Ethnische Mobilisierung und die Logik von Identitätskämpfne. Eine situationstheoretische Perspektive jenseits von „rational choice“. Unveröffentlichtes Manuskript, Humboldt-Universität zu Berlin.

Eisenstadt, S. N. (1981): Cultural traditions and political dynamics: the origins and modes of ideological politics. British Journal of Sociology, 32, 155-181.

Eisenstadt, S. N. (1982): The Axial Age: the emergence of transcendental visions and the rise of clerics. Archives Européennes de Sociologie, 23, 294-314.

Elster, J. (1989): The Cement of Society. A Study of Social Order. New York: Cambridge University Press.

Elster, J. (1991): Rationality and social norms. Archives européennes de sociologie, 32, 109129.

Emirbayer, M. (1997): Manifesto for a relational sociology. American Journal of Sociology, 103, 281-317.

Fishbein, H. D. (1976): Evolution, Development and Children's Learning. Pacific Palisades, CA: Goodyear Publication Company.

Haas, P. M. (Ed.). (1992): Epistemic Communities and International Policy Coordination. special issue of International Organization, 46, No. 1.

Habermas, J. (1979): Communication and the Evolution of Society. London: Heinemann.

Habermas, J. (1987): The Theory of Communicative Action. Lifeworld and System. A Critique of Functionalist Reason. Volume II. Boston, MA: Beacon Press.

Harris, M. (1977): Cows, Pigs, Wars, and Witches. The Riddles of Culture. London: Fontana.

Harris, M. (1978): Cannibals and Kings. The Origins of Cultures. New York: Random House.

Harris, M. (1979): Cultural Materialism: The Struggle for a Science of Culture. New York, NY: Random House.

Heiskala, R. (1997): Society as Semiosis. Helsinki: Unpublished doctoral dissertation, University of Helsinki.

Holland, J. H., Holyoak, K. J., Nisbett, R. E., \& Thagard, P. R. (1986): Induction: Processes of Inference, Learning, and Discovery. Cambridge, MA: MIT Press. 
Revista de Sociología 22 / 2008

Facultad de Ciencias Sociales - Universidad de Chile

Honneth, A. (1992): Kampf um Anerkennung. Frankfurt: Suhrkamp.

Knorr-Cetina, K. (1981): The Manufacture of Knowledge. Oxford, NY: Pergamon.

Levitt, B., \& March, J. G. (1988): Organizational learning. Annual Review of Sociology, 14,319-340.

Luhmann, N. (1995): Social Systems. Stanford, CA: Stanford University Press.

March,J. G. (1988): Decisions and Organizations. Oxford: Basil Blackwell.

March,J. G. (1991): Exploration and exploitation in organizational learning. Organization Science, 2, 71-87.

March, J. G., \& Olsen, J. P. (1984):The new institutionalism: organizational factors in political life. American Political Science Review, 78, 734-739.

March, J. G., \& Olsen, J. P. (1989): Rediscovering Institutions: The Organizational Basis of Politics. New York, NY: Free Press.

Milbrath, L.W. (1989): Envisioning a Sustainable Society: Learning OurWay Out. Buffalo, NY: State University of New York Press.

Miller, M. (1986): Kollektive Lernprozesse. Studien zur Grundlegung einer soziologischen Lerntheorie. Frankfurt: Suhrkamp.

Peters, B. (1994):Why is it so hard to change the world? International Sociology, 9, 275293.

Plotkin, H. C. (Ed.). (1982): Learning, Development, and Culture. Chichester, NY:Wiley \& Sons.

Powell, W. W., \& DiMaggio, P. J. (Eds.). (1991): The New Institutionalism in Organizational Analysis. Chicago, IL: University of Chicago Press.

Pringle, J.W. S. (1951): On the parallel between learning and evolution. Behaviour. An International Journal of Comparative Ethology, 3, 174-215.

Sabatier, P. A., \& Jenkins-Smith, H. C. (Eds.). (1993): Policy Change and Learning: An Advocacy Coalition Framework. Boulder, CO:Westview Press.

Sahlins, M. (1976): Culture and Practical Reason. Chicago, IL: University of Chicago Press.

Schmid, M., \& Wuketits, F. M. (1987): Evolutionary Theory in Social Science. Dordrecht/ Boston: Reidel.

Searle, J. (1995): The Social Construction of Reality. New York:The Free Press.

Siegenthaler, H. (1993): Regelvertrauen, Prosperität und Krisen. Tübingen: Mohr. 
Simon, H. A. (1991): Bounded rationality and organizational learning. Organization Science, 2, 125-134.

Somers, M. R. (1994): The narrative constitution of identity. Theory and Society, 23, 605-649.

Strydom, P. (1992): The ontogenetic fallacy: the immanent critique of Habermas' developmental logical theory of evolution. Theory, Culture \& Society, 9, 65-93.

Strydom, P. (1993): Sociocultural Evolution or the Social Evolution of Practical Reason: Eder's Critique of Habermas. Praxis International, 13, 304-322.

Swidler, A. (1986): Culture in action: symbols and strategies. American Sociological Review, 51, 273-286.

Taylor, C. (1992): Multiculturalism and the "Politics of Recognition". With commentary by Amy Gutman, Editor; Steven C. Rockefeller; Michael Walzer; and Susan Wolf. Princeton, NJ: Princeton University Press.

Thompson, M., Ellis, R., \& Wildavsky, A. (1990): Cultural Theory, or, Why All That is Permanent is Bias. Boulder, CO:Westview Press.

Wuthnow, R. (1987): Meaning and Moral Order: Explorations in Cultural Analysis. Berkeley, CA: University of California Press.

Wuthnow, R. (1990): Communities of Discourse. Ideology and Social Structure in the Reformation, Enlightenment, and European Socialism. Cambridge, MA: Harvard University Press.

Wynne, B. (1992): Risk and social learning: reification to engagement. In S. Krimsky \& D. Golding (Eds.), Social Theories of Risk (pp. 275-297). Westport, CT: Praeger. 